\title{
El turismo de aventura como impacto socioeconomico en el canton La Mana
}

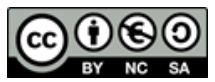

Adventure tourism as socioeconomic impact in the Canton La Mana

Yolanda Tatiana Carrasco Ruano. ${ }^{1}$

Recibido: 05-09-2018 / Revisado: 11-09-2018 / Aceptado: 22-09-2018 / Publicado: 05-10-2018

\section{Resumen. DOI: $\underline{\text { https://doi.org/10.33262/concienciadigital.v1i4.904 }}$}

El Ecuador es reconocido por practicar el turismo de aventura ya que es un pequeño paraíso por su biodiversidad y su riqueza de climas y paisajes. Famoso por sus volcanes atractivos como los Illinizas, el Cotopaxi, Cayambe y Chimborazo, y por sus caminatas entre los picos nevados de los Andes ecuatorianos, el Ecuador cuenta también con una maravillosa cultura llena de historias y leyendas.

De todas las montañas de los Andes ecuatorianos, las mejor conocidas son Cayambe $(5.789 \mathrm{~m})$, Cotopaxi $(5,897 \mathrm{~m})$ y Chimborazo $(6,310 \mathrm{~m})$. Para alcanzar estas grandes alturas una buena aclimatación es indispensable a fin de disfrutar de las ascensiones plenamente. Por esta razón ofrecemos una escuela de glaciar y caminatas por los picos más bajos, y en los días de descanso, paseos a las aguas termales de las tierras altas. En todos estos viajes será acompañado por guías profesionales que se distinguen por su constante atención a la seguridad.

Para aquellos que quieran tener una experiencia en los fascinantes paisajes de los Andes, ofrecemos una selección de excursiones de senderismo a través de las regiones más bellas de nuestro país. Si gusta de la naturaleza virgen y disfrutar de camping, considere nuestros programas de senderismo. Durante nuestras excursiones incluimos experimentados guías, transporte todo-terreno, entradas, alimentación y equipo de campamento.

Palabras claves: Cultura, Turismo, Servicios turísticos, Aventura, Economía.

\footnotetext{
${ }^{1}$ Ciencia digital, Ambato, Ecuador, tcarrascor@yahoo.com
} 


\section{Abstract.}

Ecuador is known for practicing adventure tourism since it is a small paradise due to its biodiversity and its wealth of climates and landscapes. Famous for its attractive volcanoes such as Illinizas, Cotopaxi, Cayambe and Chimborazo, and for its walks among the snow-capped peaks of the Ecuadorian Andes, Ecuador also has a wonderful culture full of stories and legends.

Of all the mountains of the Ecuadorian Andes, the best known are Cayambe $(5,789 \mathrm{~m})$, Cotopaxi $(5,897 \mathrm{~m})$ and Chimborazo $(6,310 \mathrm{~m})$. To reach these great heights a good acclimatization is essential in order to fully enjoy the ascents. For this reason we offer a glacier school and treks for the lower peaks, and on days off, trips to the hot springs of the highlands. In all these trips will be accompanied by professional guides who are distinguished by their constant attention to safety.

For those who want to have an experience in the fascinating landscapes of the Andes, we offer a selection of hiking excursions through the most beautiful regions of our country. If you like virgin nature and enjoy camping, consider our hiking programs. During our excursions we include experienced guides, all-terrain transportation, tickets, food and camping equipment. "

Keywords: Culture, Tourism, Tourist services, Adventure, Economy.

\section{Introducción.}

\section{Turismo de aventura en el mundo}

\section{Contrastes de autores}

Según los autores Gallegos G.2007, Cunin E. 2010 \& Castgnani H. 2012, el turismo de aventura en el mundo son viajes históricos que se puede situar de forma general o por giras. Estos se establecieron como una práctica basada en un hecho social de desplazamiento que reunirá principalmente finalidades políticas, culturales y de diversión.

\section{Criterio personal}

Según lo leído en el turismo de aventura en el mundo dice que se va mas en los cruceros donde los turistas realizan énfasis en donde nos permite entrar en refección porque en el mundo el turista no tiene mucha vegetación y al turismo casi se lo realiza de cruceros por varios días dependiendo de las condiciones climáticas para que el turista tenga menor riesgo en su actividad turística y también se ve la seguridad de que en cada centros que brindan turismo tenga el equipo de rescate necesario, un guía especializado y los maletines de primeros auxilios deberán tener en cuento sea el trascurso del viaje por la seguridad del visitante. 
Los turistas viajan también por saber de nueva culturas y a saber sobre su valor historia que maca cada uno de los montículos antiguos y a conocer sobre que infraestructura dotan en el país para realizar turismo de aventura.

Figura N 1. Derrame económico de turismo.

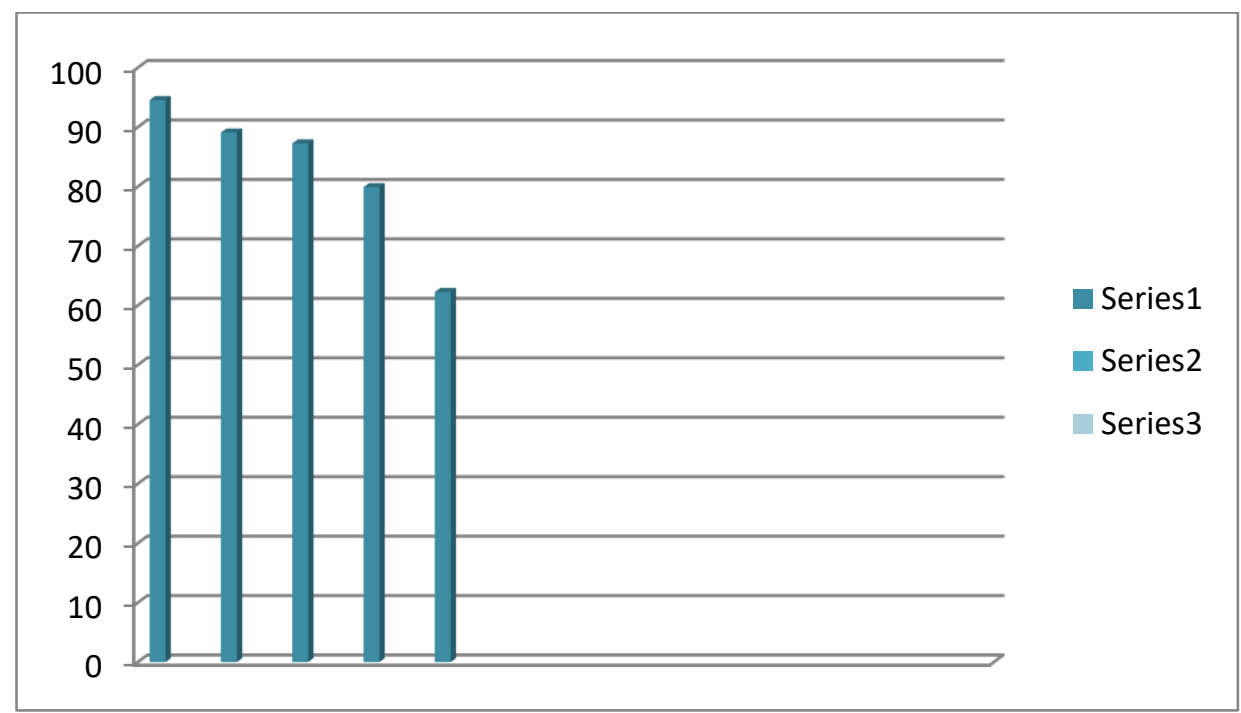

Fuente: Elaboración propia.

\section{Turismo de aventura en el ecuador}

\section{Contraste de autores}

Según los autores Cañizares C. 2015, Ramírez K. 2013 \& Villafuerte M. 2015, el turismo de aventura en el ecuador nace de la motivación de viaje del turista de sentir adrenalina mediante las actividades deportivas extremas. Esto involucra nuevas vivencias dentro de un espacio natural, y las experiencias vividas por los turistas dependerán del nivel de riesgo y de los desafíos que cada individuo pretende satisfacer, unos de los más utilizados en la actualidad y el que más ingresos económicos deja, tomando en cuenta que en el cantón La Maná se pueden practicar varios.

\section{Criterio Personal}

El turismo de aventura surge como una alternativa de diversificación de actividades para poblaciones rurales que contaban con riqueza natural, pero que no tenían los recursos necesarios para subsistir; además, es considerado como una fusión entre el culto a la naturaleza y la búsqueda de eficiencia ambiental, ya que se orienta a la conservación de los recursos naturales para convertirlos en recursos turísticos con fines lucrativos para que las personas viajen se distraigan de tanto problemas que hay en la sociedad en el ámbito laboral, con ese propósito asido creado los varios tipos de turismo en especial a donde la gente 
requiere es al turismo de aventura porque sienten la adrenalina que les brinda viajar en canoas en ríos caudalosas donde los turistas despejan su mente por un instante y solo piensa como lo va a ser y se olvidan de los problema por el entretenimiento que ellos van a tener en el ese momento no se quedarán sin satisfacer sus curiosidad de experimentar cada uno de las actividades que aran en el turismo de aventura pero este tipo de turismo debe ser controlado por lo que también tienes sus impactos negativos como es la adicción de sentir adrenalina, querer hacer lo cada rato y ahí viene la situación que el turista baja su situación económica, ya no tendrá para viajar a ciertos lugares que prestan para ese tipo de turismo y ahí es cuando el turista podría enfermarse o llegara morir pero a pesar de tan riesgo sea la actividad que lo va realizar lo hace para poder sentir la sensación, la experiencia que va a tener al momento de arriesgarse su vida por que las actividades que lo realizan lo más a menudo es el deporte de aventura por lo cual tiene una serie de desafíos que el turista desea experimentar en diferentes actividades que lo baya practicando cuando desee salir a realizarlo ya sea con amigos o familiares y al visitar los lugares lejanos que vayan visitando los turistas estarían dejando ingresos económicos para seguir avanzo o modernizando el lugar o en mejor de los casos teniendo los instrumentos para realizar deportes extremos en buen estado para cuidar mejor la vida del turista y que no sufra ningún tipo de fracturas mediante su experiencia en cualquier actividad turística activa.

Figura N 2. Porcentaje que ofrece turismo.

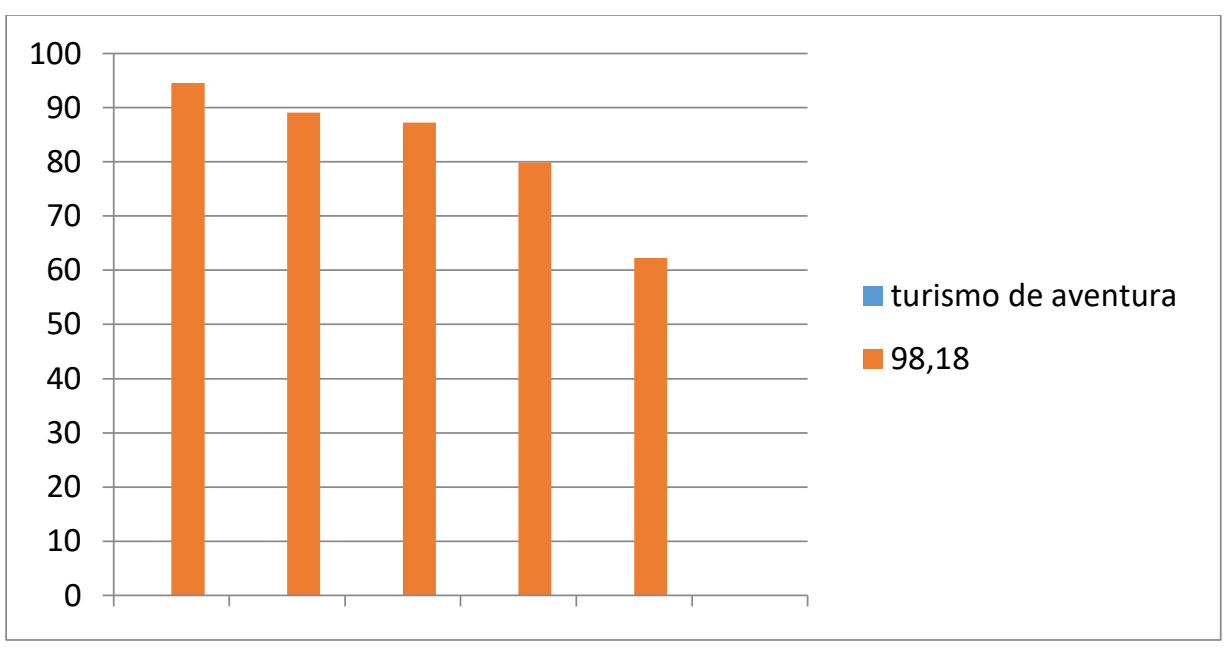

Fuente: Elaboración propia.

\section{Contraste de autores}

Según los autores Dávila C. 2013, Ecuador. 2009 \& Salazar H. 2014, el turismo de aventura se enfoca en conseguir mayor afluencia de turistas dando a conocer las diferentes actividades tales como los deportes de aventura como alternativas de recreación en diferentes lugares que practican este turismo en conjunto a su biodiversidad que lo rodea. 


\section{Criterio Personal}

Los beneficios económicos para los turistas surgen de la competitividad que se va generando al ir surgiendo cada vez más proveedores de servicios turísticos, que se esfuerzan por ganar su preferencia a través de propuestas de servicio más creativas y a mejores precios para atraer los turistas que vienen de los extranjeros, ellos requieren realizar deportes que sientan adrenalina para que el viaje de ellos sea inolvidable, por lo general en las playas es donde los turistas desean realizar estos deportes extremos donde también tienen sus riesgos y los turistas a un así quieren experimentarlos, también el turismo de aventura está relacionado con la naturaleza porque en lugares no explotados existen ríos mucho más caudalosos donde el turista podrían realizar esta actividad sin ningún problema y tendrían una ventaja por la razón que los turistas respirarían aire puro y conocerían diferentes arboles e inclusive poder conocer animales de distintas especies y aves que habitan en la naturaleza que podría ser para el turista la experiencia que haya tenido en el viaje que realizo en el impacto económico sería un poco menos por que se estaría llegan a la naturaleza donde tendría que realizar caminata, en cambio en la ciudad debe pagar un transporte para llegar al sitio turístico y poder realizar turismo y sentir la adrenalina que el turista requiere conocer sobre la actividad que realiza.

\section{Contraste de autores}

Según los autores Dionísio M. 2004, Banegas C. 2013 \& Checa N. 2013, el sistema turístico es la integración de fuerzas socioeconómicas, sociales, necesidades, requerimientos, aspectos técnicos y humanos que obliga a considerar factores inherentes al mismo, así como su entorno la actividad a llevarse a cabo, la motivación de realizarlo y el entorno en que se desarrolla, es así que, a diferencia de otras formas de recreación, el Turismo de Aventura no se centra en el sitio a ser visitado, contrario al turismo de masas, este prioriza la actividad que va a llevarse a cabo.

\section{Criterio Personal}

Los impactos económicos se originan tanto por el gasto turístico como por el desarrollo de la actividad turística (efecto multiplicador). Entre los principales impactos positivos que genera el turismo se puede mencionar su contribución a la generación de empleos. También su conexión con otros sectores empresariales, como construcción, comercio y servicios en general. Se le reconoce además su contribución al equilibrio de la balanza de pagos y al aumento y distribución de la renta.

Entre otros aspectos el turismo de aventura comprende toda la parte geográfica es donde el visitante desea realizar sus actividades de nuevos conocimientos de adrenalina es necesario en el lugar donde va a visitar tenga un alojamiento, restauración cultura y el impacto medio ambiental, el turismo de aventura es una motivación para el turista por que no se centra solo 
en el sitio que va ser visitado, al contrario el turista prioriza la actividad que va a llevarse a cabo, brindándole al turista una oportunidad de experimentar nuevas emociones, sensaciones a un nivel más alto de adrenalina que estas actividades frecuentemente se lo realiza al aire libre este turismo de aventura se comercializa por lo general en las playas con el ambiente natural y lejos del hogar del turista extranjero. La forma de entender al turismo de aventura es saber que todos los viajeros se inclinan a visitar lugares turísticos es por las vacaciones son más activas que incluyan deportes de alta peligrosidad que contenga ciertos riesgos o exploraciones en la geografía, en la naturaleza y en su entorno que la rodea.

\section{Contraste de autores}

Según los autores Herrera S. 2013, Lopez H. 2015 \& Valverde H. 2015, el adecuado uso del turismo genera ingresos al país así como el cuidado de las zonas donde los practican ya que conviven con el medio ambiente lo que es conveniente hacer una buena publicidad para difundir de mejor manera el turismo de aventura en especial con la ayuda de las autoridades.

\section{Criterio Personal}

El turismo representa una alternativa de desarrollo económico para cualquier economía; si a esto se le agrega el planeamiento y monitoreo de la actividad, puede convertirse en un importante mecanismo de conservación de los recursos naturales y culturales. Se trata además de una actividad con un importante efecto multiplicador en la economía de los países en donde se desarrolla. Precisamente al desarrollarse la actividad turística se generan una serie de impactos.

La sensación de sentir la adrenalina se lo puede practicar en varias actividades como son el senderismo interpretativo, rafting, rapel y el salto en bungee son las actividades por lo general el turista desea realizar en sus vacaciones, en el senderismo por lo general el visitante requiere conocer rutas donde divisara una flora y fauna cuyo fin específico es el conocimiento de un medio natural, en la otra actividad está el rafting contribuye a un deporte extremo donde se conjugan las destrezas y el espíritu de aventura, consiste en que el visitante deberá recorrer ríos de mucho riesgo donde el caudal va a ser fuerte y el turista lo tendrá que realizar en una balsas de goma o a su vez en canoas. El rapel es otra de las opciones que el turista desea realizar por la razón que al escalar una montaña con la respectiva seguridad el turista subirá a una altura extremada para sentir la adrenalina desde la cima de la montaña visualizará un paisaje hermoso con mucha vegetación y a dificultar que le va presentar al bajar de tan extremada altura en ese momento el turista experimenta nuevas actividades de aventura.

Otra actividad es el salto en bungge consiste en brincar de un puente a 270 pies de altura suspendido en el aire con la ayuda de un arnés y dejarse caer momentáneamente hacia un cañón con rio tropical en el fondo y la sensación de quedar suspendido a pocos metros del rio resulta ser una experiencia bastante cargada de adrenalina. 
En este turismo de aventura en cada uno de los lugares deben ofertar los bienes y servicios necesarios para que el visitante quede satisfecho del lugar escogido para sus actividades turísticas, cabe destacar que en los meses más feriados el lugar turístico tiene que estará en perfectas condiciones para poder satisfacer al visitante y también en estos feriados es la mayor afluencia de visitantes.

\section{Contraste de autores}

Según los autores Campos R. 2010, Soto A. 2015 \& Vallejo R. 2015, el turismo de aventura es uno de los más importantes conforma a la ayuda socioeconómica de las comunidades en las que se practica este deporte, ya que la actividad turística es muy importante para el uso de varios deportes turísticos, la cual ayuda a la economía del sector y al cuidado de las especies que conviven con ellos, poniendo especial cuidado en los factores de desarrollo económico, social y cultural, sin menoscabo del cuidado y preservación del ambiente.

\section{Criterio Personal}

El turismo de aventura en el cantón La Maná, practicado de manera sustentable, generará un impacto positivo en las comunidades receptoras, evitando en todo momento generar en ellas los inconvenientes que se viven en las grandes ciudades, tales como tráfico, contaminación, drogas, prostitución, violencia, delincuencia, etcétera. El turismo de aventura puede impactar si logra generar empleos que proporcionen a la población una mejor calidad de vida, y que el intercambio cultural entre propios y visitantes genere una conciencia de cohesión e identidad social, y permita a la comunidad desarrollarse generando para sus miembros inclusión, igualdad y justicia social.

Por otro lado para poder viajar y poder realizar turismo el acceso de la vía debe estar en perfectas condiciones, señalización, puentes, basureros y cabañas deben estar en buen estado para una mejor imagen de establecimiento que va ser visitado.

El turismo de aventura es uno de los segmentos que mayor crecimiento ha marcado en los últimos años, este tipo de turismo también crea fuentes de empleo para la sociedad y genera ingresos económicos, en muchas ocasiones el turismo estaba considerado como un pequeño nicho del sector turístico por la razón que era una actividad bastante difícil de catalogar pero al mismo tiempo cada vez más destinos y rutas seguían apareciendo ya sea en senderismo seguían surgiendo rutas paisajes bellos donde el turista podría apreciar la naturaleza y la hermosa flora y fauna que puede brindar cada uno de los sectores donde se practica la actividad turística ya que mediante muchos deportes turísticos ya sea en el agua, en las montañas, en caminatas y en muchas otras cosas más genera fuentes económicas para poder progresar implementando nuevas actividades que pueda servir de mucho interés al turista ya que el visitante desea experimentas cosas diferentes y así tener mejor ingreso y satisfacción 
del turista para así que el lugar no sea abandonado y sea mejor acogido mejor por los visitantes y el lugar con mejor infraestructura.

\section{Conclusiones}

- El cantón La Maná es un paraíso de escenarios naturales por lo que se concluye que es un excelente lugar para los turistas ya que considera que es un lugar muy tranquilo para conocer nuevos paisajes naturales y sobretodo ser partícipe de un turismo de aventura.

- La infraestructura turística con que cuenta el cantón La Maná es importante para poder desarrollarse como producto turístico. Por lo que este cantón si cuenta con todo lo necesario para formar parte de los lugares más turísticos del país y realizar deportes de aventura.

- Es primordial que la gestión municipal sea más activa en el sector ya que se considera que el turismo en esta zona es el factor socioeconómico más importante que posee, además de brindar las facilidades necesarias en cuanto a servicios básicos, planta e infraestructura turística.

- No se ha dado una planificación adecuada para convertir al cantón en lugar pleno y exitosamente turístico, desperdiciando de esta manera la riqueza natural que no ha sido explotada de un modo beneficioso.

\section{Referencias bibliográficas.}

Alvarez, R. (2013). El turismo de aventura practicado de manera sustentable. Ambato, Ecuador.

Banegas, C. (2013). El turismo de aventura en la sociedad. Quito, Ecuador.: ESPE.

Campos, R. (2010). Lugares turísticos del cantón La Maná. Latacunga, Ecuador.: ESPE.

Cañizares, C. (2015). La actividad turística en el Ecuador. Quito, Ecuador: Universidad San Francisco de Quito.

Castgnani, H. (2012). La sociología del cuerpo.

Castignani, G. G. (s.f.). Las actividades físico-deportivas en la naturaleza y la industria turistica. 
Checa, N. (2013). El turismo de aventura, interacción con el ambiente natural. Quito, Ecuador.

Cunin, E. (2010). Turismo Globalización y Alteridad.

Davila, C. (2013). Turismo de aventura en el país. Guayaquil, Ecuador.

Dionísio, M. (2004). El sistema turístico. Quito, Ecuador.: ESPE.

Ecuador, C. d. (2009). Reglamento del Ministerio de Turismo del Ecuador. Quito, Ecuador.

Gallegos, G. (2007). Las actividades físico- deportivas en la naturaleza y la industria turística.

Gonzalez, S. (2015). Actividades del impacto socioeconómico en el turismo. Latacunga, Ecuador.: Ecotravel.

Herrera, S. (2013). Los tipos de turismo de aventura. Quito, Ecuador.: ESPE.

Lopez, H. (2015). Los servicios turísticos que ofrece el cantón La Maná. Latacunga, Ecuador.

Ramirez, K. (2013). Lugares turísticos de la provincia de Cotopaxi. Latacunga, Ecuador.

Salazar, H. (2014). El turismo de aventura en conjunto a la naturaleza. Quito, Ecuador.

Soto, A. (2015). El turismo de aventura como influencia del sector turístico. Latacunga, Ecuador.

Vallejo, R. (2012). La naturaleza en convivencia del turismo de aventura. Quito, Ecuador.: ESPE.

Valverde, H. (2015). El impacto socioeconómico en el turismo de aventura. Quito, Ecuador.

Villafuerte, M. (2015). La actividad turística en el cantón La Maná. Ambato, Ecuador.

Viteri, M. (2010). Los impactos económicos en las actividades turísticas. Quito, Ecuador.

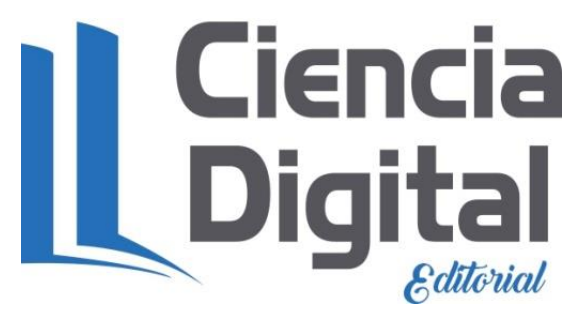


Para citar el artículo indexado.

Carrasco Ruano, Y. (2018). El turismo de aventura como impacto socioeconomico en el canton La Mana. ConcienciaDigital, 1(4), 24-33.

https://doi.org/10.33262/concienciadigital.v1i4.904

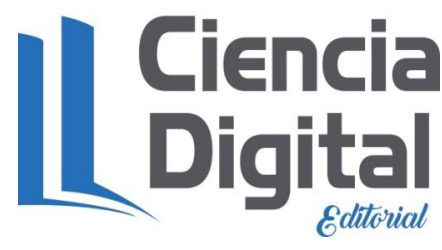

El artículo que se publica es de exclusiva responsabilidad de los autores y no necesariamente reflejan el pensamiento de la Revista Conciencia Digital.

El articulo queda en propiedad de la revista y, por tanto, su publicación parcial y/o total en otro medio tiene que ser autorizado por el director de la Revista Conciencia Digital.
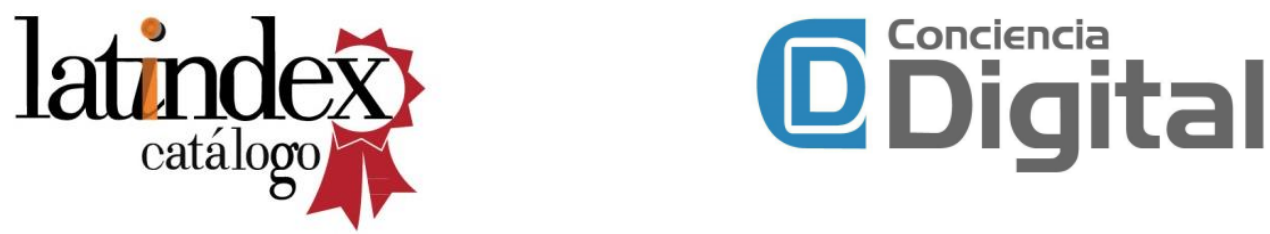